This is the author's final, peer-reviewed manuscript as accepted for publication. The publisher-formatted version may be available through the publisher's web site or your institution's library.

\title{
Remote non-invasive assessment of pain and health status in cattle
}

Miles E. Theurer, David E. Amrine, Brad J. White

\section{How to cite this manuscript}

If you make reference to this version of the manuscript, use the following information:

Theurer, M. E., Amrine, D. E., \& White, B. J. (2013). Remote non-invasive assessment of pain and health status in cattle. Retrieved from http://krex.ksu.edu

\section{Published Version Information}

Citation: Theurer, M. E., Amrine, D. E., \& White, B. J. (2013). Remote noninvasive assessment of pain and health status in cattle. Veterinary Clinics of North America: Food Animal Practice, 29(1), 59-74.

Copyright: Copyright (C) 2013 Elsevier Inc.

Digital Object Identifier (DOI): doi:10.1016/j.cvfa.2012.11.011

Publisher's Link: http://www.sciencedirect.com/science/article/pii/S0749072012000990

This item was retrieved from the K-State Research Exchange (K-REx), the institutional repository of Kansas State University. K-REx is available at http://krex.ksu.edu 


\section{Remote non-invasive assessment of pain and health status in cattle}

Miles E. Theurer, BS ${ }^{\mathrm{a}}$; David E. Amrine, DVM ${ }^{\mathrm{a}}$; Brad J. White, DVM, MS ${ }^{\mathrm{b}}$

${ }^{a}$ Department of Diagnostic Medicine and Pathobiology, Kansas State University, 1800 Denison

Avenue, Manhattan, KS 66506

${ }^{\mathrm{b}}$ Department of Clinical Sciences, Kansas State University, 1800 Denison Avenue, Manhattan, KS 66506

The authors have nothing to disclose.

*Corresponding author for proof and reprints:

Brad J. White, DVM, MS

Department of Clinical Sciences, Kansas State University

Mosier Hall Q 211

1800 Denison Avenue, Manhattan, KS 66506

Phone: (785) 532-4243

Fax: (785) 532-4989

bwhite@vet.k-state.edu (email)

Coauthors addresses:

Miles E. Theurer

Department of Diagnostic Medicine and Pathobiology, Kansas State University

Mosier Hall J 118

1800 Denison Avenue, Manhattan, KS 66506

Phone: (785) 532-2895

Fax: (785) 532-4989

mtheurer@vet.k-state.edu (email)

David E. Amrine, DVM

Department of Diagnostic Medicine and Pathobiology, Kansas State University Mosier Hall I 111

1800 Denison Avenue, Manhattan, KS 66506

Phone: (785) 532-4803

Fax: (785) 532-4989

damrine@vet.k-state.edu (email)

Keywords: behavior monitoring, remote sampling, animal welfare 


\section{Synopsis}

Cattle behavior is frequently monitored to determine health and wellness state of the animal. The objective of this review is to describe potential benefits and challenges of remotely monitoring cattle behavior with available methodologies including clinical illness scores, visual monitoring, accelerometers, pedometers, feed intake and behavioral monitoring, global position systems, real time location systems, thermography images, and rumen telemetry temperature bolus. The behavior of interest, labor required, and monitoring expenses all need to be taken into consideration before deciding which remote behavioral monitoring device is most appropriate. Monitoring the feeding behavior of an animal over a period of time allows establishment of a baseline against which deviations in subsequent behavioral patterns can be evaluated. Interpretation of multiple behavioral responses as an aggregate indicator of animal wellness status rather than as individual outcomes may be a more accurate measure of true state of animal pain or wellness status.

\section{Key Points}

- Cattle behavior is frequently monitored to determine health and wellness state.

- Available remote monitoring systems include:

- clinical illness scores

- visual monitoring

- accelerometers

- pedometers

- feed intake and behavioral monitoring

- global position systems 
- real time location systems

- thermography images

- rumen telemetry temperature bolus

- Selection of remote behavioral monitoring system is influenced by:

- the behavior of interest(frequency and type)

- labor required to monitor the animals

- monitoring expenses

- Interpretation of multiple behavioral responses as an aggregate indicator of animal wellness status rather than as individual outcomes may be a more accurate measure of true state of animal pain or wellness status.

\section{Introduction}

The ability to remotely identify cattle that require an intervention due to pain or disease is important for animal health providers and researchers. Behavior is frequently monitored to measure potential changes in animal well-being. ${ }^{1}$ Stress, pain, or disease may alter animal behavior relative to optimal wellness status, but monitoring these changes is challenging without a clear definition of the expected behavioral response to an adverse event. ${ }^{2}$ Some behavioral definitions are vague, and they are not specifically tied to one pain or disease response. Improvement in behavioral monitoring techniques is needed for remote monitoring of activity to be useful as a diagnostic or research tools.

Multiple methods are available to monitor cattle behavior including subjective visual observation, objective measures of cattle activity, or determination of cattle location within the housing area. Subjective measurements of pain and cattle well-being include behavioral, depression, or illness scores based on observer impression of the animal's current wellness state. 
The challenges with utilizing subjective measures to determine cattle wellness state are related to potential differences both between observers and among observers over time.

An opportunity exists to more discretely identify potential behavioral changes via collection of data utilizing remote sensing technologies. Objective, continuous behavioral monitoring using accelerometers and pedometers has been used to assess cattle behavior in a variety of scenarios..$^{3-9}$ Monitoring cattle location within a defined environment has also been used in an effort to identify and monitor potential behavioral changes. ${ }^{8,10}$

The objective of this review is to describe potential benefits and challenges of remotely monitoring cattle behavior with available methodologies including clinical illness scores, visual monitoring, accelerometers, pedometers, feed intake and behavioral monitoring, global position systems, and real time location systems. Although all of these remote monitoring systems are not directly applicable in a clinical setting, the results from research based on these technologies provides valuable insights to practitioners on the associations between behavioral changes and pain and wellness states.

\section{Observer monitoring clinical illness}

One of the most common methods to determine wellness or painful state of an animal is having a trained observer monitor cattle for clinical signs of pain or disease. Multiple clinical signs and subjective assessments can be used to determine the animal's overall wellness status. Often a combination of findings can be categorized into a single value, or clinical illness score (CIS), which represents the current state of the animal. The potential benefit of determining a CIS is presumably that it correlates with the need for an intervention or the probability of a specific outcome. ${ }^{11}$ Scoring systems that assign a value based on degrees of illness are relatively common, ${ }^{12}$ and are frequently used in disease research. ${ }^{3,10,13}$ Even when quantitative 
measurements, such as rectal temperature, are combined with subjective assessment, the final disease classification remains subjective. ${ }^{14,15}$ This subjectivity may impact how the results are interpreted if the CIS is used as one of the criteria in a treatment or preventative health program.

Research has shown very limited agreement among observers using the same CIS to identify calves with respiratory disease. ${ }^{16}$ Potential sources of variation include differences among the experience and training of observers, cattle type, and environmental conditions. When a subjective scoring system is applied and interpreted by more than one individual, it should be repeatable among those individuals. Others have evaluated agreement among veterinarians assigning body condition scores to cows and determined even small amounts of training among the observers can increase the overall agreement. ${ }^{17} \mathrm{~A}$ clear case definition and educational programs can decrease the variation between observers and make the results more clinically applicable.

Although CIS are frequently utilized, true accuracy relative to disease state is difficult to determine. There is no gold standard to diagnose respiratory disease in cattle, but the presence or absence of pulmonary lesions at harvest has been compared with ante mortem diagnoses of clinical respiratory disease. ${ }^{18-20}$ Results from these studies illustrate low correlations between lung scores and diagnosis of clinical illness. White and Renter ${ }^{21}$ estimated the sensitivity and specificity of using clinical signs of illness combined with rectal temperature to diagnose respiratory disease to be $61.8 \%$ and $62.8 \%$, respectively. A test with imperfect sensitivity and specificity can underestimate or overestimate morbidity, thus leading to errors in the interpretation of preventative or therapeutic treatment efficacy. ${ }^{16}$

One way to improve CIS agreement among observers is the implementation of a refined scoring system with limited categories. The objective of assigning CIS to cattle is to accurately

\section{Page 5}


identify those animals which need an intervention (sensitivity) and those that would not (specificity); therefore, the system could be condensed to those two categories. If calves are deemed to require an intervention, the selection of the intervention would be based on clinician's judgment of the case. For example, a calf that was deemed to have clinical respiratory disease may require an intervention with an antimicrobial; while euthanasia may be a more appropriate intervention for an animal severely ill enough they become moribund and non-responsive to human approach. Dichotomizing the results would help agreement among observers and could potentially increase accuracy of comparison of CIS among individual observers, as previous research has illustrated distinguishing illness severity based on CIS is challenging. ${ }^{10,16}$ Much of the analysis of CIS data is based on the dichotomization of an animal into healthy or sick, therefore systems that have more than two main levels serve limited purpose.

Monitoring clinical illness by visual appraisal is a common procedure and the specific implementation of the scoring system influences final data interpretation. Although CIS are quantitative, they may not be repeatable between or among observers and do not provide an objective measure of the degree of clinical illness. Care should be taken to limit potential sources of variability among observers through training and selection of the appropriate scoring system for the situation.

\section{Observer monitoring frequency of specific behavior}

Comparing calf wellness status among treatment groups in a research environment or over time in a clinical application can also be performed by monitoring the frequency of specific behaviors associated with pain or disease. Researchers have noted increase in specific behaviors such as the number of head shakes, ear twitches, and foot stomps after a painful procedure such 
as castration. ${ }^{22-24}$ Other researchers have documented a difference in head shakes and ear twitches following dehorning. ${ }^{25-29}$

The frequency of all these behaviors has been associated with increased cortisol concentrations, and increased cortisol concentrations are often associated with stress and fearful events. $^{30,31}$ However, neither cortisol nor counts of these behavior measurements have been determined as specific indicators of pain. Calves may increase counts of ear flicks, tail switches, and foot stomps following painful procedures, but these behaviors may also increase with high insect burden. ${ }^{32,33}$ These behavioral counts are not specific for pain or wellness status, but they are cost effective and relatively easy to obtain through live observations or video analysis.

The most cost effective method to determine the frequency of these behaviors is having an observer document the activities as they occur in the field. A limitation of this method is that behavioral activities occurring at rapid rates (e.g. ear flicks) can be challenging to accurately record as they occur. ${ }^{34}$ In a population environment, recording these behaviors on more than one animal simultaneously can also be challenging. Cattle activity may also be difficult to interpret when the observer is in close enough proximity to document specific behaviors, as studies have shown the presence of a human observer to alter cattle behavior. ${ }^{35,36}$ Cattle behavioral patterns change throughout the day following a circadian rhythm, ${ }^{37}$ but it is difficult for an observer to continuously document cattle behavior for 24 hours a day. Due to these limitations, monitoring of these behaviors is commonly performed through video analysis.

The use of the video collection technology allows observers to analyze cattle behavior at their leisure and enables observation of tail flicks, ear twitches, stomping, postural behavior, and positional location just as collected during live observation. Video recording systems are relatively easy to set up and can be used in most scenarios. The output files can be viewed on a 
variety of common electronic devices including laptop computers and DVD players, allowing for minimal additional input costs to view the videos. The required quality of the video recording system is based on the specific behavior desired to document, the number of animals to observe, and environmental conditions.

Limitations of video analysis include the need to clearly identify and visualize individual animal activity, as well as the labor required to document the frequency of specific behaviors. Identification of individual animals is important to document while observing multiple animals in a pen level setting when the experimental unit is the individual, but identification on the video may be difficult using only a visual identification ear tag or coat coloration patterns. Animals may be uniquely marked with all-weather paint sticks, spray paint, and hair dye to ease the ability to identify animals on video, but all of these markings will wear away making it necessary to apply multiple times. Determining frequency of behavioral counts is difficult in low ambient light, but adding artificial lights has shown to increase the amount of time dairy cows spend lying down and reduced distance traveled. ${ }^{38}$ Depth perception is decreased while watching video footage compared to live observation that can make it challenging to determine if animals are actually eating or drinking or just spending time near the feeder or water. Another issue with video observation is the labor involved to view and document all the behavior activity desired.

Video viewing is a time consuming and tedious task. Software exists to make data recording of animal behavior easier for the viewer while watching the video. ${ }^{39,40}$ Video sampling methods have been evaluated to reduce the amount of labor required to analyze the video. Continuous, scan, time, and focal animal sampling have all been used to minimize the labor required to classify segments of video, yet still accurately determine animal behavior. 
Continuous sampling is observing animal activity for the entire period that data were captured at the same speed that video was recorded. Scan sampling is observing animal behavior for a brief period, then repeating the observation after a period of time. ${ }^{41}$ The portion of time passed between recording samples is the scan interval and is set at a pre-determined length. The frequency of behavioral activity monitored during the observation period is used to represent the percentage of behavior activity over the entire period of time. ${ }^{42}$ Scan sampling has been shown to accurately evaluate frequency of cattle behaviors compared to continuous sampling, but when the scan interval was $\geq 30$ minutes (a 30 minute gap between sampling periods), correlation to continuous sampling decreased. ${ }^{43}$

Time sampling is identifying behavior for a period of 10 minutes at the beginning of each hour and then multiplying the frequency of behavioral activity by 6 to represent activity for the entire hour. ${ }^{44}$ Time sampling has low correlation coefficients compared to continuous monitoring for describing standing, lying, feeding, drinking, and walking activity. ${ }^{43}$ This low correlation makes time sampling a less accurate method for classifying cattle behaviors based on recorded video.

Focal sampling is the monitoring of a portion of animals within the group for the entire period to determine behavioral activity for the group. Focal sampling of 1 animal out of 10 animals per pen was accurate for describing all 10 animals standing, lying, feeding and walking behaviors; however watering behavior required observing 4 out of 10 animals per pen to accurately describe drinking behavior. ${ }^{43}$ Individual animal variation in the behaviors of interest may influence accuracy of focal sampling, but this technique may be appropriate for some pen level studies. Observing video clips at the rate of 4 times faster than recorded speed has 
accurately depicted swine feeding and watering behavior in confined settings compared to realtime recording speed. ${ }^{44}$

Video recording and documenting counts of specific behaviors can be used to monitor potential changes related to pain or wellness status; however, the process is time and labor intensive. The use of scan and focal sampling will reduce the amount of labor required to accurately determine animal behavior. Despite potential limitations, continuous video monitoring is considered as the "gold standard" by which other behavior monitoring devices are evaluated.

\section{Monitoring activity with accelerometers}

Accelerometers are devices that continuously measure gravitational force in multiple axes, and these values can be processed to determine activity and postural behaviors. Figures 1A and $1 \mathrm{~B}$ show a three-dimensional accelerometer attached with the horizontal, vertical, and diagonal axes the accelerometer monitors gravitational force. Before remote continuous monitoring technology can be used to assess the physiological and behavioral patterns cattle display, the technology requires validation. ${ }^{45,46}$ Accelerometers have been shown to accurately monitor calf behaviors of standing, lying, or walking with $97.7 \%$ agreement to video analysis. ${ }^{6}$ This high accuracy allows the user to effectively rely on the accelerometers to determine posture behavior rather than using a labor intensive process of analyzing video.

Assessing postural changes may be important in evaluating calf wellness or pain status, and several studies have illustrated differences in postural behavior following painful stimuli. Calves have been shown to increase the percentage of time standing in the hours immediately following castration based on accelerometer analysis. ${ }^{47}$ However, Pauly et al. determined calves spent more time lying down and less time walking in the five day period following castration. ${ }^{5}$ The difference between these two studies may be due to the length of the monitoring period and 
a potential time dependent change in behaviors. Theurer et al. determined calves administered the non-steroidal anti-inflammatory drug, meloxicam, prior to cautery dehorning spent more time lying down for 5 days post-dehorning compared to control calves that did not receive analgesia as commonly performed in production practice. ${ }^{8,48}$ Lying behavior decreased in calves after being induced with experimental lameness using an amphotericin B synovitis-arthritis induction model. ${ }^{49}$ Accelerometers are an effective tool for continuous monitoring of behavior changes in response to pain.

Accelerometers (GP1 SENSR, Reference LLC, Elkader, IA) have also been used to monitor disease and wellness state of cattle. Calves challenged with Mannheimia haemolytica spent more time lying down compared to unchallenged control calves. ${ }^{7}$ This agrees with a common assumption that a primary clinical sign of respiratory disease is depression. In another respiratory disease trial, there was no difference in the amount of time morbid calves spent lying down or walking compared with baseline data collected prior to challenge. ${ }^{3}$ These findings suggest that the postural activity of cattle may be influenced by disease or pain state, but changes in standing and lying behavior may not be a specific response to changes in wellness status.

Daily environmental conditions, differences among individual calves, and circadian rhythms also affect the amount of time calves spend lying, ${ }^{37}$ therefore, it is important to make comparisons of behavioral activities to calves housed in the same environmental conditions. Monitoring control animals allows the observer to distinguish between the behavioral change associated with administering a procedure from daily variation due to environmental conditions. ${ }^{37,50,51}$ The placement of the accelerometer on the animal and accelerometer size and

\section{Page 11}


weight may transiently alter normal gait and behavior. A brief acclimation period may be needed for the cattle to adjust to having the accelerometer attached to their leg.

Limitations of using accelerometers to monitor behavior include cost, data processing, and technological constraints. Accelerometers are relatively expensive compared to other behavior monitoring techniques, such as video analysis. Transforming the accelerometer into useable behavioral measurements can be achieved with validated algorithms; however, generating the data processing technique is time consuming. The accelerometers must have sufficient battery life, on board memory storage (or the ability to wirelessly transmit data), and be small enough to be easily affixed to the animal in some method. The objective quantification of cattle postural behavior as determined by accelerometers provides valid data to compare potential changes in behavioral patterns associated with pain or wellness status.

\section{Monitoring step count frequency with pedometers}

Pedometers have been used to objectively quantify the number of steps traveled and total distance traveled. An on-board algorithm calculating the number of steps from the raw data is contained within the pedometer. Pedometers are relatively easy to attach and use, but the number of steps each calf travels varies considerably among days and environmental conditions.

The distance calves travel may be associated with painful and stressful procedures. The amount calves travel following a painful procedure such as castration may vary as some research demonstrated calves traveled fewer steps for 4 days after castration; $; 2$ while other work was unable to detect a difference in the number of steps traveled in calves after castration. ${ }^{53}$ Stress may also influence the distance traveled as calves have been shown to take more steps for 3 days after weaning. ${ }^{54}$ Bulls travel more steps than steers per day indicating the need for accounting for 
gender in the analysis. ${ }^{52}$ In properly designed experiments, pedometers may be useful in determining changes in behavior following a painful procedure.

Pedometers have been used to detect early lameness in dairy cattle, but a $15 \%$ decrease in activity was needed before the pedometer could accurately identify $92 \%$ of lame cattle. ${ }^{55}$ The biological significance of a $15 \%$ decrease in activity has not been established, but there may be clinical implications in detecting cattle before a change this large is detected. O'Callaghan et al. demonstrated lame dairy cows traveled 22.5 fewer steps per hour compared to non-lame cows based on visual locomotion score throughout the majority of days into milk. ${ }^{56}$ As pedometers are directly measuring locomotion, they are a valuable tool in identifying and monitoring musculoskeletal pain. However, changes in step counts as measured by pedometers are not specific for only identifying pain as the use of the pedometer technology has been able to accurately detect the onset of estrus in cows due to increase activity levels. ${ }^{57,58}$

Pedometers can be an effective monitoring device for evaluating pain response and health status of cattle. The relative lower cost of investment and labor intensity compared to other technologies makes pedometers an attractive tool to objectively monitor potential behavioral changes.

\section{Feed intake and behavioral monitoring}

Systems are available to measure individual cattle feeding behavior and intake in group housed situations. These systems have been used to identifying morbid cattle from healthy cattle based on differences in feeding behaviors. ${ }^{59}$ Feed and water intake, duration, and frequency are specific behaviors that can be monitored with these systems. Systems that monitor feeding and watering behaviors that are commercially available include GrowSafe (GrowSafe Systems Ltd, Airdrie, AB Canada) and Insentec (Repelweg, Marknesse, Netherlands). GrowSafe utilizes radio 
frequency identification (RFID) ear tags to identify individual animals. Insentec on the other hand uses transponder collars to identify when animals are at feeding or watering stations. Both systems have integrated software that allows for real-time monitoring and analysis of animal feeding or watering behavior.

Radio frequency identification technology has been used to document a reduction in the frequency of visits to feeders. ${ }^{24}$ Researchers evaluating residual feed intake (RFI) found distinct differences in feeding behaviors among high and low RFI calves using both the GrowSafe and Insentec monitoring systems. ${ }^{60,61}$ Since feed inputs represent one of the largest costs in producing beef, monitoring behaviors that may identify calves with less than ideal feed efficiencies may be beneficial. ${ }^{62}$ Monitoring the feeding behavior of an animal over a period of time allows establishment of a baseline against which deviations in subsequent behavioral patterns can be evaluated. Investigators have used algorithms with 7 day rolling average feeding times as baselines to identify behavioral changes correlated with painful locomotive conditions in dairy cows days before farm staff were able to diagnose lameness. ${ }^{63}$

Monitoring animal feeding behavior and intake can provide insight into potential changes in wellness or pain status. Setup, maintenance, training, and expense are all potential disadvantages that must be considered when evaluating remote feed intake and behavior systems. However, the feed intake and frequency data collection capabilities make these systems an attractive monitoring tool to use since feed costs are important to the producer.

\section{Location determination: global positioning systems (GPS)}

Global positioning systems (GPS) have been used to remotely monitor movement of wildlife and domestic animals. ${ }^{64,65}$ Advances in GPS technology have created lighter and more accurate receivers, but monitoring multiple animals in varied geographic regions is often cost 
prohibitive. ${ }^{65}$ Three of the largest challenges when monitoring cattle with GPS technology are the ability to have real time updates, decreased battery life, and spatial accuracy.

Current technology allows for the location of a GPS receiver to be updated every second, but this update rate exceeds the power sources available in most animal monitoring units. ${ }^{66}$ Custom units with real time updates once every minute have been developed. However, battery life was only 3.7 days. ${ }^{67}$ Others using non-real time receivers have successfully monitored cattle for longer durations (11 days) by only waking the system up from a deep sleep mode every 600 seconds; however, depending on the environment these infrequent readings may not provide the level of data necessary to define specific behaviors. ${ }^{68}$

Positional accuracy of the systems are also an issue and some research shows a discrepancy between visual and tag position of an average \pm standard deviation of $9 \mathrm{~m} \pm 7 \mathrm{~m} .{ }^{69}$ Other work illustrates that $99.9 \%$ of positional fixes fell within $20 \mathrm{~m}$ and $97.3 \%$ within $10 \mathrm{~m}$ of a known point. ${ }^{68}$ Based on these accuracies, the GPS can give approximate location of individuals, but readings are not discrete enough to delineate specific activities such as eating or drinking.

The tradeoffs of battery life and positional update frequency limit the potential uses of GPS systems in situations where the behavior needs to be continually monitored for longer periods of time. Accuracy of $10 \mathrm{~m}$ may be sufficient for questions of pasture usage and grazing activities, but is not sufficient for monitoring feeding and watering behaviors. These limitations make GPS difficult to use to monitor changes in pain or wellness status in cattle.

\section{Monitoring movement in a defined system with real-time location systems (RTLS)}

Real-time location systems (RTLS) are designed to locate the position of an item anywhere within a defined area. The architecture of an RTLS consists of receivers spaced around the desired monitoring space, active or passive tags which are placed on the objects one wants to 
monitor, computer hardware, and software to receive and translate positional data. Tags used with most RTLS systems are smaller and have considerably longer battery life than current GPS technology. Like GPS, most RTLS requires line of sight from tags to sensors for accurate readings. Figure 2 demonstrates a calf within the sensor area and shows how 3 of receivers locate the animal and triangulate its position. Amount of time is calculated by subtracting the time of arrival at that location from the previous time of arrival documented. While similar to RFID behavior and intake systems, RTLS has the distinct advantage of being able to monitor an animal's location anywhere within the pen thus not restricting evaluation to only feeding and drinking behaviors.

The system monitors location within the pen at pre-set time intervals and not the specific behavior the calf is engaged in while at that position. Therefore, for data to be useful, the positions must be matched with a known diagram of the facility structure with specific areas of interest (proximity to feed, water, shelter) identified on the same scale of axes as measured by the RTLS system. Depending on the frequency of measurements, the RTLS can be used to document the percent of time animals spend in specific locations within the housing environment.

An advantage of RTLS is the ability to measure levels of activity such as distance traveled and time spent within a given proximity to other calves. By measuring location over discrete time intervals, the data can be compared to determine the distance an animal traveled over a given period with results similar to measurements taken using pedometers. Social interactions with other calves (or the lack thereof) can be monitored by comparisons the proximity of individual calves to other animals within the pen. Real-time location systems have 
been used to monitor potential changes in cattle behavior that may be associated with pain or alterations in wellness status.

Investigators have used RTLS technology (Ubisense, Denver, CO) to determine that certain behaviors, such as time spent at the feed bunk and distance traveled, were associated with clinical illness scores. ${ }^{10}$ The distance traveled by calves as monitored with RTLS was also associated with the level of lung consolidation, indicating that monitoring movement may be a reasonable tool for wellness status evaluation. ${ }^{10}$ Theurer et al. identified calves that were dehorned and given pain medication had different feeding behaviors as measured by RTLS technology compared to calves dehorned without pain medications. ${ }^{8}$ These associations with behavior changes indicate RTLS technology is a valid tool to generate quantitative measurements of cattle activities that can be used to monitor potential changes in wellness or pain status in response to an intervention.

Limitations of RTLS technology include expense and technological constraints. The RTLS systems are able to monitor animal behavior within a specific area, but those areas need to be equipped with multiple sensors to accurately monitor behavioral activity which may be cost prohibitive in many situations. Installation and calibration of a RTLS requires significant investment in time and resources and while the use of this technology for monitoring animals is relatively new, these systems have been used successfully for many years monitoring assets in large complex manufacturing environments.

\section{Thermography images}

Thermography imaging can be used to monitor and record surface temperatures in multiple species. This technology has been used to non-invasively monitor welfare in cattle $\mathrm{e}^{70}$ and monitor nasal mucosal temperatures. ${ }^{71}$ Using thermography in cattle housed in high ambient

\section{Page 17}


temperatures has been shown to result in low sensitivity $(70.7 \%)$ and adequate specificity (89.5\%) of identifying animals above or below a rectal temperature cutoff value. ${ }^{72}$

Corneal surface temperatures have been monitored using thermography based on the hypothesis that changes in corneal temperature may be reflective of changes in core temperature resulting from pain or disease. In one study there was no difference in surface temperature 2-3 hours after dehorning procedure. ${ }^{73}$ Maximum surface corneal temperature has been shown to decrease $0.27^{\circ} \mathrm{C}$ from baseline 2-5 minutes in calves post disbudding without local anesthesia. ${ }^{74}$ However cattle disbudded had higher surface temperature 5-15 minutes after disbudding compared to controls that were not disbudded. ${ }^{74}$ Temporal relationships need to be taken into account when analyzing thermography images.

Cattle infected with foot-and-mouth disease virus have been monitored using infrared thermography imaging; however, this system resulted in a low sensitivity $(61.1 \%)$ and adequate specificity (87.7\%) of correctly identifying infected animals with foot-and-mouth disease. ${ }^{75}$ There was not a strong correlation between face surface temperature and rectal temperature, but there was a positive correlation between foot surface temperature and rectal temperature indicating peripheral extremities may be more illustrative of core body temperature. The use of infrared thermography imaging has also resulted in low sensitivity (67.6\%) and adequate specificity (86.8\%) of identifying calves with bovine respiratory disease. ${ }^{76}$

Thermography images can be relatively easy to capture, but in order to capture the images correctly the distance from the camera and the animal needs to be relatively consistent. Environmental conditions impact the relative temperatures recorded on images and need to be standardized to collect images for comparison when designing a research trial. Interpretation of the thermography images needs to include the temporal relationship related to the procedure 
performed (establishment of a baseline reading in similar environmental conditions) in order to detect changes. Thermography imaging needs to have refinement of the cutoff values used to detect morbid or painful animals before becoming implemented into industry practice.

\section{Rumen telemetry temperature bolus}

Rumen telemetry temperature boluses have been used to non-invasively monitor the health parameters of cattle. ${ }^{77}$ Rumen temperatures have been shown to increase in calves challenged with Mannheimia haemolytica, and rumen temperatures have also been shown to have a strong correlation $\left(R^{2}=0.80\right)$ to rectal temperatures. ${ }^{78}$ The use of reticulo-rumen boluses has a positive predictive value of $73 \%$ for identifying animals infected with BRD when compared to a physical exam. ${ }^{79}$ The pyrogenic effect of lipopolysaccharide was shown to only transiently increase rectal temperatures when administered to dairy calves, ${ }^{51}$ however rumen temperature increased $2{ }^{\circ} \mathrm{C}$ when administered lipopolysaccharide to beef heifers. ${ }^{77}$

Limitations of rumen bolus telemetry system include the expense and administration of the bolus into the animal. The continued automatic thermal documentation has potential advantages related to remote monitoring of temperature changes. Overall effectiveness of the the rumen telemetry temperature bolus is impacted by specific facets of the system including: ability to use telemetry in the specific environment (interference, geographic distribution), data collection and management plan.

\section{Tympanic bulla and intravaginal temperature monitors}

Tympanic bulla temperature can be monitored using a portable data logger attached to a thermistor. Temperature readings obtained with tympanic bulla thermometers have been

correlated to rectal temperature, ${ }^{80,81}$ and tympanic temperature has been shown to increase 0.78 and $0.65{ }^{\circ} \mathrm{C}$ by moving cattle around in the summer and winter respectively. ${ }^{82}$ The increase in 
body temperature due to processing needs to also be taken into consideration when evaluating the health status of an individual animal.

Intravaginal temperature can be monitored using a thermistor modified with finger-like projections in order to prevent expulsion from the vagina. ${ }^{57}$ The onset of estrus has been able to be determined using intravaginal temperature moinitors. ${ }^{57,83}$ However, there is little published literature describing the use of tympanic bulla and intravaginal temperature monitors to determine health or wellness states.

\section{Summary}

Determining animal wellness status is frequently based on visual appraisal or performance parameters. The use of multimodal, remote, quantitative monitoring techniques will become more critical in determining the physiological, behavioral, and performance responses cattle experience in different scenarios. Interpretation of multiple behavioral responses as an aggregate indicator of animal wellness status rather than as individual outcomes may be a more accurate measure of true state of well-being. Individual animals differ greatly in behavior and accurate interpretation of behavioral changes is dependent on the ability to establish normal baseline activity in calves in a specific housing environment.

Behavioral data should be interpreted carefully as none of the commonly monitored behaviors are truly specific for one type of illness or pain response. Statistical analyses should account for the hierarchy of repeated measures on individual calves, the effect of having multiple observers, housing effects, time of day, and seasonality. If these potential sources of variability are not included in statistical analysis, differences between treatment groups may be falsely detected or there may be differences that are undetected. 
There are numerous remote monitoring methods available to assess the pain or well-being status of an animal; however determination of the specific behavior needed to monitor, labor, and expense all need to be taken into consideration before deciding which behavioral monitoring device to use. The selection of the appropriate system for the situation is dependent on the expected benefits compared to costs of operating the system. Utilizing remote monitoring system provides basic information on cattle behavioral changes that can be translated to other aspects of clinical practice and animal wellness evaluation.

\section{References}

1. Gonyou HW. Why the study of animal behavior is associated with the animal welfare issue. J Anim Sci. 1994;72(8):2171-2177.

2. Levitis D, Lidlicker WZ, Freund G. Behavioural biologists do not agree on what constitutes behaviour. Anim Behav. 2009;78(1):103-110.

3. Hanzlicek GA, White BJ, Mosier DA, et al. Serial evaluation of physiologic, pathological, and behavioral changes related to disease progression of experimentally induced Mannheimia haemolytica pneumonia in postweaned calves. Am J Vet Res. 2010;71(3):359-369.

4. Theurer ME, White BJ, Anderson DE, et al. Effect of transportation during periods of high ambient temperature on physiology and behavior of beef heifers. Am J Vet Res. Accepted, In Press.

5. Pauly C, White BJ, Coetzee JF, et al. Evaluation of analgesic protocol effect on calf behavior after concurrent castration and dehorning. Int J Appl Res Vet M. 2012;10(1):5461. 
6. Robert B, White BJ, Renter DG, et al. Evaluation of three-dimensional accelerometers to monitor and classify behavior patterns in cattle. Comput Electron Agr. 2009;67(1-2):8084.

7. Theurer ME, Anderson DE, White BJ, et al. Effect of Mannheimia haemolytica pneumonia on behavior and physiologic responses of calves experiencing hyperthermal environmental conditions Paper presented at: XXVII World Buiatrics Conference 2012; June 3-8, 2012, 2012; Lisbon, Portugal.

8. Theurer ME, White BJ, Coetzee JF, et al. Assessment of behavioral changes associated with oral meloxicam administration at time of dehorning in calves using a remote triangulation device and accelerometers. BMC Vet Res. 2012;8(1):48.

9. Dockweiler J, Bergamasco L, Coetzee J, et al. Effect of age and castration method on physiological stress indicators in calves. Paper presented at: Phi Zeta Sigma Chapter, 2012; Manhattan, Kansas.

10. White BJ, Anderson DE, Renter DG, et al. Clinical, behavioral, and pulmonary changes following Mycoplasma bovis challenge in calves. Am J Vet Res. 2012;73(4):490.

11. Hayes G, Mathews K, Kruth S, et al. Illness severity scores in veterinary medicine: what can we learn? J Vet Intern Med. 2010;24(3):457.

12. Perino LJ, Apley M. Clinical trial design in feedlots. Vet Clin N Am-Food A. 1998;14(3):243-266.

13. Coetzee JF, Edwards LN, Mosher RA, et al. Effect of oral meloxicam on health and performance of beef steers relative to bulls castrated on arrival at the feedlot. J Anim Sci. 2012;90(3):1026. 
14. Sanderson MW. Designing and running clinical trials on farms. Vet Clin N Am-Food A. Mar 2006;22(1):103-123.

15. Wenz JR, Garry FB, Barrington GM. Comparison of disease severity scoring systems for dairy cattle with acute coliform mastitis. J Am Vet Med Assoc. 2006;229(2):259.

16. Amrine DE, White BJ, Larson RL, et al. Determining precision and accuracy of clinical illness scores compared to pulmonary consolidation scores in Holstein calves with induced Mycoplasma bovis pneumonia. Am J Vet Res. Accepted, in press.

17. Kristensen E, Dueholm L, Vink D, et al. Within- and across-person uniformity of body condition scoring in Danish Holstein cattle. J Dairy Sci. 2006;89(9):3721-3728.

18. Wittum TE, Woollen NE, Perino LJ, et al. Relationships among treatment for respiratory tract disease, pulmonary lesions evident at slaughter, and rate of weight gain in feedlot cattle. J Am Vet Med Assoc. 1996;209(4):814-818.

19. Schneider MJ, Tait RGJ, Busby WD. An evaluation of bovine respiratory disease complex in feedlot cattle: Impact on performance and carcass traits using treatment records and lung lesion scores. J Anim Sci. 2009;87(5):1821-1827.

20. Thompson PN, Stone A, Schultheiss WA. Use of treatment records and lung lesion scoring to estimate the effect of respiratory disease on growth during early and late finishing periods in South African feedlot cattle. J Anim Sci. 2006;84(2):488-498.

21. White BJ, Renter DG. Bayesian estimation of the performance of using clinical observations and harvest lung lesions for diagnosing bovine respiratory disease in postweaned beef calves. J Vet Diagn Invest. 2009;21(4):446-453.

22. Robertson IS, Kent JE, Molony V. Effect of different methods of castration on behaviour and plasma cortisol in calves of three ages. Res Vet Sci. 1994;56(1):8-17. 
23. Mellor DJ. Effects of castration on behaviour and plasma cortisol concentrations in young lambs, kids and calves. Res Vet Sci. 1991;51(2):149.

24. Gonzalez LA, Schwartzkopf-Genswein KS, Caulkett NA, et al. Pain mitigation following band castration of beef calves and its effects on performance, behavior, E. coli, and salivary cortisol. J Anim Sci. 2009;88:802 - 810.

25. Morisse JP, Cotte JP, Huonnic D. Effect of dehorning on behaviour and plasma cortisol responses in young calves. Appl Anim Behav Sci. 1995;43(4):239-247.

26. McMeekan C, Stafford K, Mellor D, et al. Effects of a local anaesthetic and a nonsteroidal anti-inflammatory analgesic on the behavioural responses of calves to dehorning. New Zeal Vet J. 1999;47(3):92-96.

27. Graf B. Behavioural and physiological responses of calves to dehorning by heat cauterization with or without local anaesthesia. Appl Anim Behav Sci. 1999;62(2-3):153.

28. Stilwell G, Carvalho RC, Carolino N, et al. Effect of hot-iron disbudding on behaviour and plasma cortisol of calves sedated with xylazine. Res Vet Sci. 2010;88(1):188-193.

29. Vickers KJ, Niel L, Kiehlbauch LM, et al. Calf Response to Caustic Paste and Hot-Iron Dehorning Using Sedation With and Without Local Anesthetic. J Dairy Sci. 2005;88(4):1454-1459.

30. Grandin T. Assessment of stress during handling and transport. J. Anim Sci. 1997;75(1):249-257.

31. Mormede P, Soissons J, Bluthe RM, et al. Effect of transportation on blood serum composition, disease incidence, and production traits in young calves. Influence of the journey duration. Ann Rech Vet. 1982;13(4):369-384. 
32. Hillerton JE, Bramley AJ. Variability between Muscidae populations of dairy heifers on two different types of pasture in southern England. Brit Vet J. 1986;142(2):155-162.

33. Harris JA, Hillerton JE, Morant SV. Effect on milk production of controlling muscid flies, and reducing fly-avoidance behaviour, by the use of fenvalerate ear tags during the dry period. J Dairy Res. 1987;54(2):165-171.

34. Altmann J. Observational study of behavior: sampling methods. Behaviour. 1974;49(3):227.

35. Ishiwata T, Kilgour RJ, Uetake K, et al. Choice of attractive conditions by beef cattle in a Y-maze just after release from restraint. J Anim Sci. 2006;85(4):1080.

36. Grignard L. The social environment influences the behavioural responses of beef cattle to handling. Appl Anim Behav Sci. 2000;68(1):1.

37. Robért BD, White BJ, Renter DG, et al. Determination of normal beef cattle activity patterns utilizing wireless accelerometers: circadian rhythms, variation among days, and differences between individual calves. Am J Vet Res. 2011;72(4):467.

38. Phillips CJC, Schofield SA. The effect of supplementary light on the production and behaviour of dairy cows. Anim Prod. 1989;48(2):293-303.

39. Hänninen L. CowLog: Open-source software for coding behaviors from digital video. Behav Res Methods. 2009;41(2):472.

40. Morrow-Tesch JL, Dailey JW, Jiang H. A video data base system for studying animal behavior. J Anim Sci. 1998;76(10):2605.

41. Mitlohner FM, Morrow-Tesch JL, Wilson SC, et al. Behavioral sampling techniques for feedlot cattle. J Anim Sci. 2001;79(5):1189.

42. Colgan PW. Quantitative ethology: Wiley; 1978. 
43. Mitlohner FM, Morrow-Tesch JL, Wilson SC, et al. Behavioral sampling techniques for feedlot cattle. J. Anim Sci. May 1, 2001 2001;79(5):1189-1193.

44. Arnold-Meeks C, McGlone JJ. Validating techniques to sample behavior of confined, young pigs. Appl Anim Behav Sci. 1986;16(2):149.

45. Duff GC, Galyean ML. Board-invited review: recent advances in management of highly stressed, newly received feedlot cattle. J Anim Sci. 2007;85(3):823-840.

46. Weary DM, Huzzey, J.M., von Keyserlingk, M.A.G. Board-Invited Review: Using behavior to predict and identify ill health in animals. J. Anim Sci. 2009;87:770-777.

47. White BJ, Coetzee JF, Renter DG, et al. Evaluation of two-dimensional accelerometers to monitor behavior of beef calves after castration. Am J Vet Res. 2008;69(8):1005-1012.

48. Coetzee J, Nutsch A, Barbur L, et al. A survey of castration methods and associated livestock management practices performed by bovine veterinarians in the United States. BMC Vet Res. 2010;6(1):12.

49. Schulz KL, Anderson DE, Coetzee JF, et al. Effect of flunixin meglumine on the amelioration of lameness in dairy steers with amphotericin B-induced transient synovitisarthritis. Am J Vet Res. 2011;72(11):1431.

50. Fuquay JW. Heat stress as it affects animal production. J Anim Sci. 1981;52(1):164.

51. Theurer ME, Anderson DE, White BJ, et al. Physiological and behavioral changes with variations in ambient temperature and exposure to lipopolysaccharides in cattle. Paper presented at: American Association of Bovine Practitioners, 2011; St. Louis, Missouri.

52. Devant M, Marti S, Bach A. Effects of castration on eating pattern and physical activity of Holstein bulls fed high-concentrate rations under commercial conditions. J Anim Sci. 2012. 
53. Currah JM, Hendrick SH, Stookey JM. The behavioral assessment and alleviation of pain associated with castration in beef calves treated with flunixin meglumine and caudal lidocaine epidural anesthesia with epinephrine. Can Vet J. 2009;50(4):375-382.

54. Haley DB, Bailey DW, Stookey JM. The effects of weaning beef calves in two stages on their behavior and growth rate. J. Anim Sci. 2005;83(9):2205-2214.

55. Mazrier H, Tal S, Aizinbud E, et al. A field investigation of the use of the pedometer for the early detection of lameness in cattle. Can Vet J. 2006;47(9):883-886.

56. O'Callaghan KA, Cripps PJ, Downham DY, et al. Subjective and objective assessment of pain and discomfort due to lameness in dairy cattle. Anim Welfare. 2003;12(4):605.

57. Redden KD, Kennedy AD, Ingalls JR, et al. Detection of estrus by radiotelemetric monitoring of vaginal and ear skin temperature and pedometer measurements of activity $J$ Dairy Sci. 1992;76:713-721.

58. Roelofs JB, van Eerdenburg FJ, Soede NM, et al. Pedometer readings for estrous detection and as predictor for time of ovulation in dairy cattle. Theriogenology. Nov 2005;64(8):1690-1703.

59. Sowell BF, Branine ME, Bowman JG, et al. Feeding and watering behavior of healthy and morbid steers in a commercial feedlot. J Anim Sci. 1999;77(5):1105-1112.

60. Nkrumah JD, Basarab JA, Price MA, et al. Different measures of energetic efficiency and their phenotypic relationships with growth, feed intake, and ultrasound and carcass merit in hybrid cattle. J Anim Sci. 2004;82(8):2451.

61. Kelly AK, McGee M, Crews DHJ, et al. Effect of divergence in residual feed intake on feeding behavior, blood metabolic variables, and body composition traits in growing beef heifers. J Anim Sci. 2010;88(1):109. 
62. Bingham GM, Friend TH, Lancaster PA, et al. Relationship between feeding behavior and residual feed intake in growing Brangus heifers. J Anim Sci. Aug 2009;87(8):26852689.

63. Gonzalez LA, Tolkamp BJ, Coffey MP, et al. Changes in feeding behavior as possible indicators for the automatic monitoring of health disorders in dairy cows. J Dairy Sci. Mar 2008;91(3):1017-1028.

64. Moen R, Pastor J, Cohen Y. Effects of animal activity on GPS telemetry location attempts. Alces. 2001;37(1):207-216.

65. Davis JD, Darr MJ, Xin H, et al. Development of a GPS herd activity and well-being kit (GPS HAWK) to monitor cattle behavior and the effect of sample interval on travel distance. Appl Eng Agric. 2011;27(1):143-150.

66. Tomkiewicz SM, Fuller MR, Kie JG, et al. Global positioning system and associated technologies in animal behaviour and ecological research. Philos T Roy Soc B. 2010;365(1550):2163.

67. Schleppe JB, Lachapelle G, Booker CW, et al. Challenges in the design of a GNSS ear tag for feedlot cattle. Comput Electron Agr. 2010;70(1):84-95.

68. Trotter MG, Lamb DW, Hinch GN, et al. Global navigation satellite system livestock tracking: system development and data interpretation. Anim Prod Sci. 2010;50(6):616.

69. Schleppe JB, Lachapelle G, Booker CW, et al. Challenges in the design of a GNSS ear tag for feedlot cattle. Computers and Electronics in Agriculture. 2010;70(1):84-95.

70. Stewart M, Webster JR, Schaefer AL, et al. Infrared thermography as a non-invasive tool to study animal welfare. Anim Welfare. 2005;14:319-325. 
71. Willatt DJ. Continuous infrared thermometry of the nasal mucosa. Rhinology. 1993;31(2):63-67.

72. Gomez A, Vergara C, Cook NB, et al. Is Thermography a Possible New Method to Evaluate Body Temperature in Fresh Cows? Paper presented at: American Association of Bovine Practitioners, 2011; St. Louis, Missouri.

73. Stewart M, Stookey JM, Stafford KJ, et al. Effects of local anesthetic and a nonsteroidal antiinflammatory drug on pain responses of dairy calves to hot-iron dehorning. J Dairy Sci. 2009;92(4):1512-1519.

74. Stewart M. Eye temperature and heart rate variability of calves disbudded with or without local anaesthetic. Physiol Behav. 2008;93(4-5):789.

75. Rainwater Lovett K. Detection of foot-and-mouth disease virus infected cattle using infrared thermography. Vet J. 2009;180(3):317.

76. Schaefer AL, Cook NJ, Church JS, et al. The use of infrared thermography as an early indicator of bovine respiratory disease complex in calves. Res Vet Sci. 2007.

77. Small JA. Core body temperature monitoring with passive transponder boluses in beef heifers. Can Vet J. 2008;88(2):225.

78. Rose Dye TK. Rumen temperature change monitored with remote rumen temperature boluses after challenges with bovine viral diarrhea virus and Mannheimia haemolytica. $J$ Anim Sci. 2010;89(4):1193.

79. Timsit E. Early detection of bovine respiratory disease in young bulls using reticulorumen temperature boluses. Vet J. 2011;190(1):136.

80. Davis MS, Mader TL, Holt SM, et al. Strategies to reduce feedlot cattle heat stress: effects on tympanic temperature. J Anim Sci. Mar 2003;81(3):649-661. 
81. Mader TL, Holt SM, Hahn GL, et al. Feeding strategies for managing heat load in feedlot cattle. J Anim Sci. 2002;80(9):2373-2382.

82. Mader T, Davis M, Kreikemeier W. Case study: Tympanic temperature and behavior associated with moving feedlot cattle. Prof Anim Sci. 2005;21:339-344.

83. Rorie RW, Bilby TR, Lester TD. Application of electronic estrus detection technologies to reproductive management of cattle. Theriogenology. Jan 1 2002;57(1):137-148. 
Figures

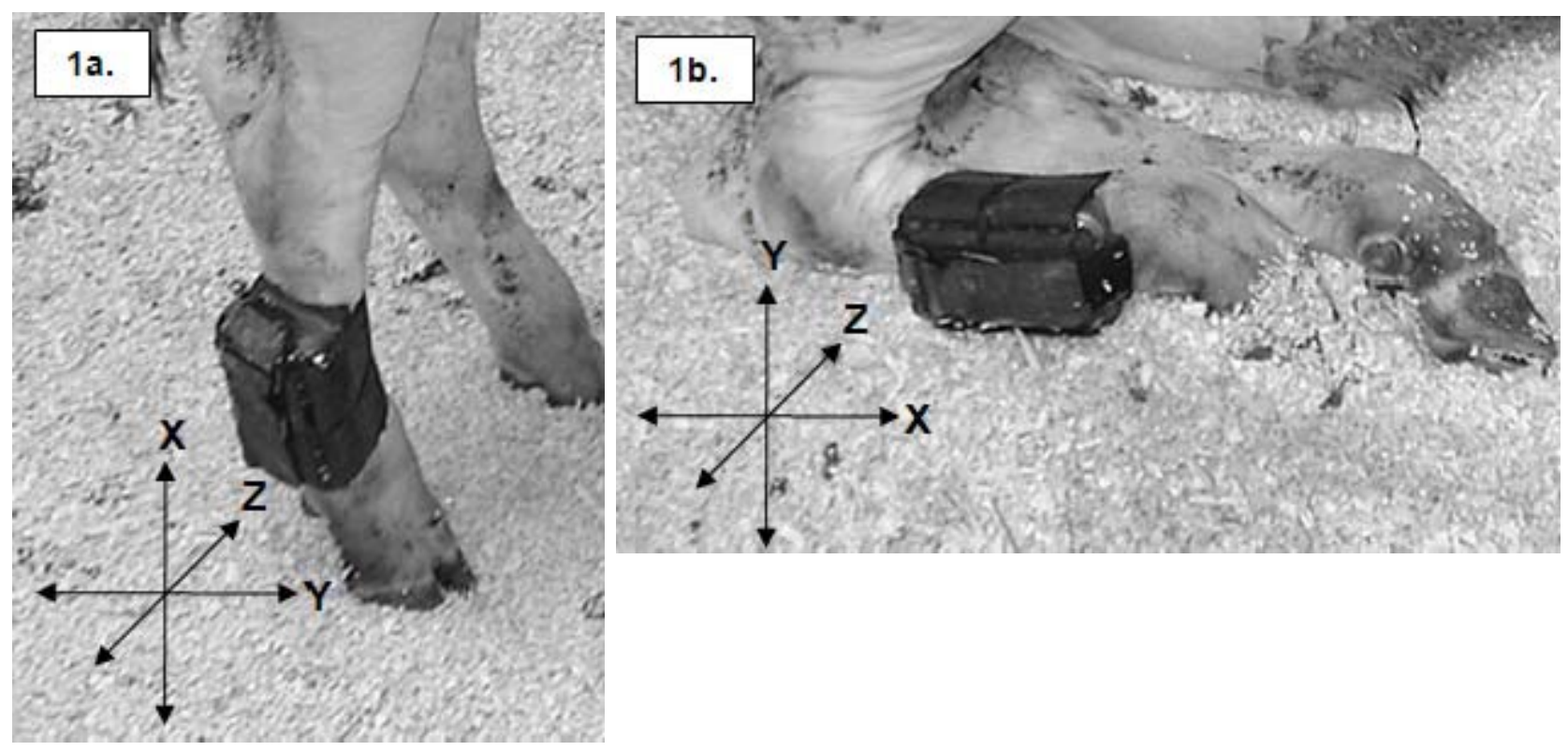

Figure 1A and 1B. Position of the three-dimensional accelerometer (and illustration of measured $\mathrm{X}, \mathrm{Y}$, and $\mathrm{Z}$ axes) on the lateral aspect of the right rear limb in a standing (1A) and lying (1B) calf. Borrowed from Robert B, White BJ, Renter DG, et al. Evaluation of three-dimensional accelerometers to monitor and classify behavior patterns in cattle. Comput Electron Agr. 2009;67(1-2):80-84, with permission.

\section{Page 31}




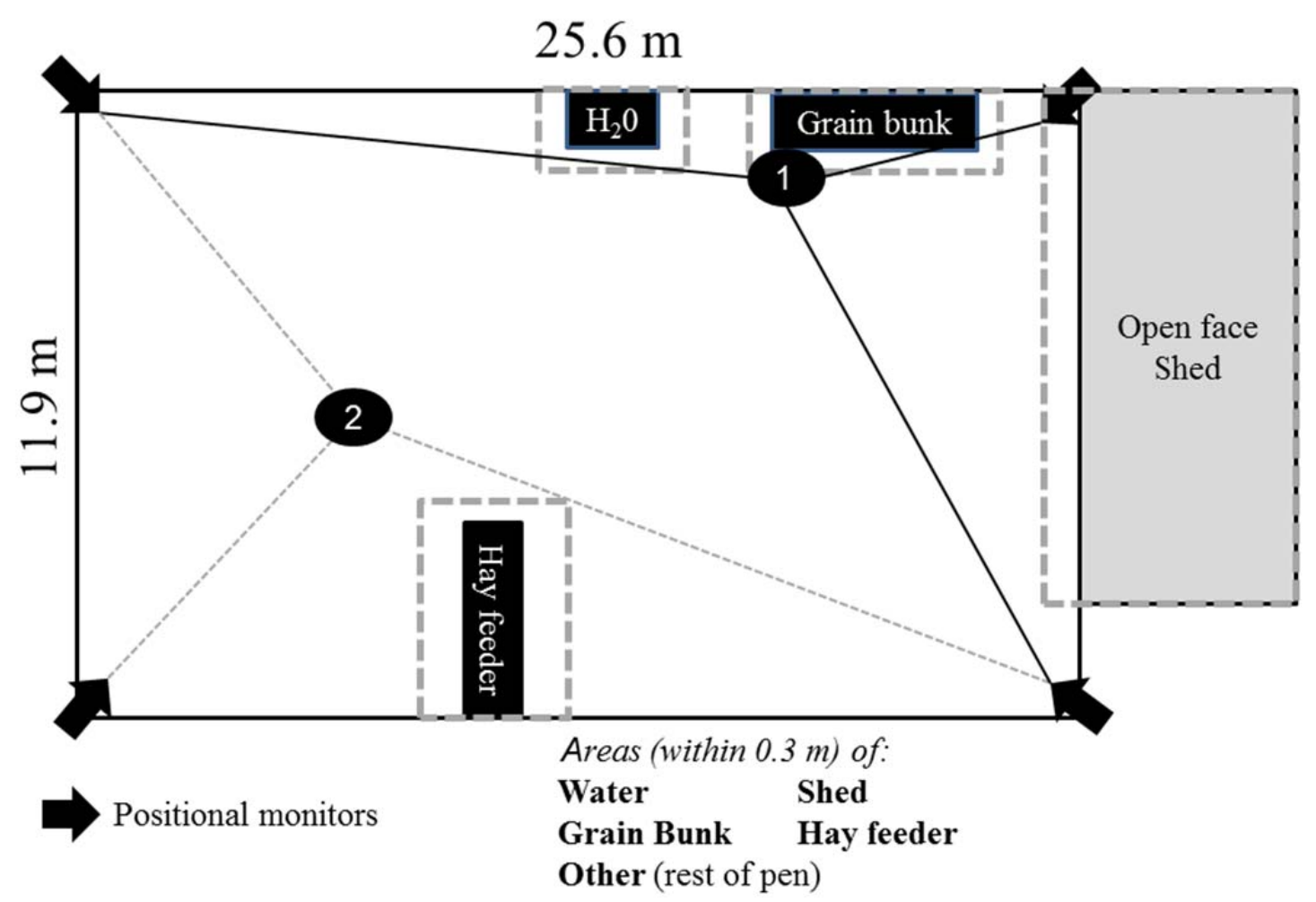

Figure 2. Stylized representation of a remote triangulation system with positional monitors (represented by arrows) able to triangulate animal position and compare to marked areas of interest including grain bunk, hay feeder, shed, and water. Calf position is determined by the relative distance between the calf tag and at least three readers (represented by lines from the readers to the points within the pen). Amount of time at a location is determined by calculating the difference between time of arrival at that specific coordinates and previous triangulation time point. Circle 1 represents a calf that would be classified as being at the grain bunk, and Circle 2 represents a calf that would be classified as in the pen, but not next to a location of interest. Adapted from Theurer ME, White BJ, Coetzee JF, et al. Assessment of behavioral changes associated with oral meloxicam administration at time of dehorning in calves using a remote triangulation device and accelerometers. BMC Vet Res. 2012;8(1):48, with permission. 